\title{
STIFFNESS AND STRENGTH-BASED LIGHTWEIGHT DESIGN OF TRUSS STRUCTURES USING MULTI-MATERIAL TOPOLOGY OPTIMIZATION
}

\author{
CLÁUdIA J. ALMEIDA ${ }^{*}$, FÁBIO M. CONDE ${ }^{\dagger}$, PEDRO G. COELHO ${ }^{\dagger}$ AND TIAGO \\ L. PRATAS ${ }^{\dagger \dagger}$ \\ ${ }^{*} \dagger$ UNIDEMI, FCT - Universidade Nova de Lisboa \\ 2829-516 Caparica, Portugal \\ e-mail: \{csj.almeida@campus.fct.unl.pt; f.conde@campus.fct.unl.pt; pgc@ fct.unl.pt\} \\ $\dagger$ IDMEC, IST - Universidade de Lisboa \\ Av. Rovisco Pais 1, 1049-001 Lisboa, Portugal \\ e-mail: \{f.conde@campus.fct.unl.pt\} \\ ${ }^{\dagger \dagger}$ EMBRAER Portugal - Estruturas Metálicas S.A. \\ Parque da Indústria Aeronáutica de Évora, 7005-797 Évora, Portugal \\ e-mail: \{tlpratas@gmail.com\}
}

Key words: Topology; Optimization; Multi-material; Stress; Truss; Compliance.

\begin{abstract}
Stiffness and strength are important structural design criteria. However, most contributions to Topology Optimization (TO) deal with the compliance minimization problem. Controlling stresses in a structure is very important to avoid material failure, but that raises complications in TO, such as: nonlinearity, singularity and high computational cost. The total weight of a structure is also another important criterion in optimal design. The multi-material setting is considered in the present work as it opens the possibility to improve structural performance even further allowing extra weight reduction. Recursive SIMP is used as the material interpolation scheme and design solutions are sought using the ground structure approach. This means that truss-like (lattice) designs are obtained here. The problem is relaxed to the continuum by introducing an artificial density variable and it is solved by a gradientbased algorithm (MMA). A stress-constraint relaxation technique ( $q p$-approach) is applied to overcome the stress singularity phenomenon. A continuation approach is used to guarantee discrete solutions, i.e., only the presence or absence of bars is identified. Therefore, design uniformity in terms of bars cross section areas is ensured. Hence, this work proposes a methodology to perform Multi-Material Topology Optimization (MMTO) of truss structures, with density-based design variables, and subject to stress constraints. To discuss the differences between stiffness and strength-oriented optimal designs, a compliance minimization problem subject to mass constraint is also considered. The example chosen demonstrates the viability of the proposed design methodology and it also reveals differences between the strongest and the stiffest designs.
\end{abstract}




\section{INTRODUCTION}

Recent developments in engineering and technology along with increasing environmental awareness have demanded more efficient solutions. Topology Optimization (TO) can be used to find improved designs in terms of weight, stiffness, and strength. The TO concept was first introduced in 1870 by Maxwell in his pioneer study [1]. Later, in 1964, Dorn et al. [2] introduced the Ground Structure Approach to optimize truss structures, where a discrete design domain is used.

TO is a discrete problem by nature. It determines, within a given design domain, either discrete or continuous, which points should be void or solid. Such problem can be solved by gradient-free optimization methods such as meta-heuristics or deterministic methods as branchand-bound $[3,4]$. Gradient-based methods can also be used provided that the original TO problem is relaxed to the continuum, becoming thus differentiable [5]. The density-based methods are one of the most common approaches to attain such relaxation. The pioneer study of Bendsøe and Kikuchi [6] introduced for the first time an artificial density variable to analyze structures made of porous material microstructures. In 1989, Bendsøe [7] proposed the Solid Isotropic Material with Penalization (SIMP) model, one of the most popular density-based approaches to solve TO problems.

In Single Material Topology Optimization (SMTO), truss design domains have been used to find design solutions in the aerospace and automotive fields (see e.g. [8,9]). Recently, MultiMaterial Topology Optimization (MMTO) has started to raise interest due to its capability to

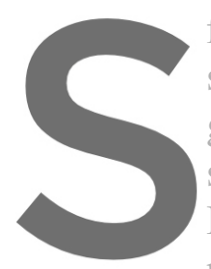
find better solutions with respect to (w.r.t.)
strength. Density-based approaches have been
generalized number of material phases, using
so-called recursive SIMP [10,11], or the Discr
Besides density-based approaches, the levelproblems [13,14].
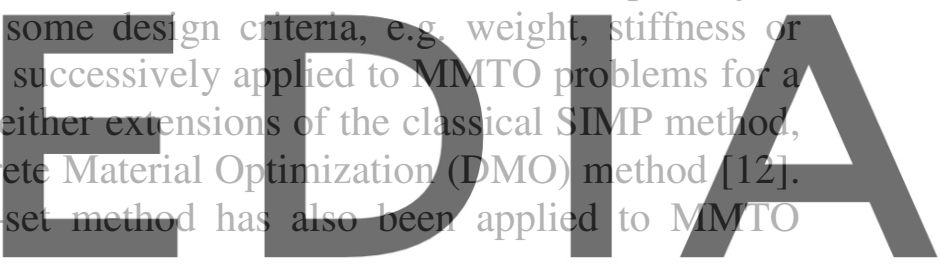

Recently, Li and Kim [15]. and Jung et al. [16], presented similar MMTO problems aiming

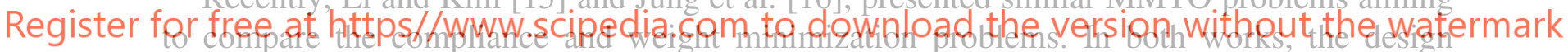

domain is discretized with plane or hexahedral elements (continuous domain). In fact, most

IVIVITO contributions deal with continuous design domains. Oniy a few works focus on muitimaterial discrete structures (lattices/trusses), see e.g. [17-22]. Some of these studies use discrete variables while others use continuous variables. Continuous variables allow the problem to be solved using gradient-based algorithms, typically quite faster than gradient-free algorithms. There are not many contributions on truss TO with the density variable, the multi-material setting and stress constraints. The present work aims to contribute in these aspects.

In the case of single-load and single-material problems, uniform material properties, no side constraints on design variables (e.g. areas), and under the isostaticity condition, the compliance and stress-based TO problems have the same optimum. In contrast, in the multi-load or multimaterial case, the optimal solution is not trivial, as there is no equivalence between the compliance and stress problems [23]. Therefore, even though most studies on TO focus on stiffness problems, rather than considering stress constraints, this work highlights the importance of imposing admissible stresses, as they are important in engineering practice [24].

The implementation of stress constraints represents a major challenge mainly due to: (1) the local nature of stress constraints; (2) non-linearity of the stress function and (3) singularity 
phenomenon. The local nature of the stress constraints, assuming a design domain discretized with finite elements, means having as many of such constraints as the number of finite elements. For problems with a large number of elements one might consider using parallel computing or constraint aggregation techniques, like the $p$-norm, to reduce the computational cost. The singularity phenomenon, as shown in [25], occurs when an optimum design is singular, i.e., it is located in a degenerated point of the design domain, unreachable by gradient-based optimization algorithms. To overcome this difficulty, relaxation techniques can be applied to stress constraints, such as the $\varepsilon$-relaxation [26,27] and the $q p$-approach [28]. The later is used in the present work.

This work is outlined as follows. The multi-material interpolation scheme used is presented in Section 2. The optimization problem formulation is presented in Section 3. In Section 4 a numerical example is presented, to prove the benefits of performing strength-oriented MMTO. Finally, Section 5 outlines the conclusions of this work and highlights the differences between stiffness and strength-oriented optimal designs.

\section{MULTI-MATERIAL INTERPOLATION SCHEME}

Each truss bar element has a material chosen from a predefined list of materials, with distinct properties of volumetric mass density $\rho_{e}$, Young's modulus $E_{e}$ and yield stress $\bar{\sigma}_{e}$. To determinate which material properties are attributed to each bar element, a multi-material interpolation scheme based on the density design variable is implemented. The well-known
SIMP method is used here in its recursive form to interpolate among $n$ different phases, i.e.,
\[ \chi_{e}\left(x_{e, 1}, x_{e, 2}, \ldots, x_{e, n-1}\right)=\sum_{m=1}^{n}\left[1-\left(x_{e, m}^{p}-x_{e, m}^{p} \delta_{m, n}\right)\right)\left(\prod_{q=1}^{m-1}\left[\left(x_{e, q}\right)^{p}\right) \chi^{(n-m+1)}\right] \]

where $x_{e, m}$ is the density variable $m$ of element $e$ (total of $n-1$ variables per element), $\chi_{e}$

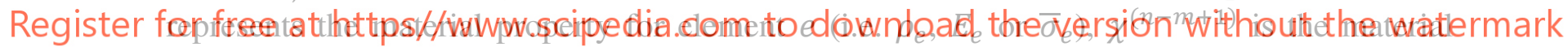
property of phase $(n-m+1), \delta_{m, n}$ is the Kronecker delta $\left(\delta_{m, n}=1\right.$ if $m=n ; \delta_{m, n}=0$ if $m \neq$ $n$ ) and the exponent $p$ imposes a penalization on intermediate densities.

In the classical Single Material Topology Optimization (SMTO) problem there are just two phases to interpolate $(n=2)$, i.e., void and solid phases. Therefore, only one design variable is required in (1), and it gives:

$$
\chi_{e}\left(x_{e, 1}\right)=x_{e, 1}^{p} \chi^{1}+\left(1-x_{e, 1}^{p}\right) \chi^{2}
$$

where $\chi^{1}=\chi^{\text {mat } 1}$ and $\chi^{2}=\chi^{\text {void }} \cong 0$.

For Multi-Material Topology Optimization (MMTO), scheme (1) is written, in this work, for three and four phases (one of them is void) as seen in (3) and (4), respectively:

$$
\begin{gathered}
\chi_{e}\left(x_{e, 1}, x_{e, 2}\right)=x_{e, 1}^{p}\left(x_{e, 2}^{p} \chi^{1}+\left(1-x_{e, 2}^{p}\right) \chi^{2}\right)+\left(1-x_{e, 1}^{p}\right) \chi^{3} \\
\chi_{e}\left(x_{e, 1}, x_{e, 2}, x_{e, 3}\right)=x_{e, 1}^{p}\left(x_{e, 2}^{p}\left(x_{e, 3}^{p} \chi^{1}+\left(1-x_{e, 3}^{p}\right) \chi^{2}\right)+\left(1-x_{e, 2}^{p}\right) \chi^{3}\right)+\left(1-x_{e, 1}^{p}\right) \chi^{4}
\end{gathered}
$$

Consider that $\chi^{3}$ and $\chi^{4}$ correspond to the void phase in (3) and (4), respectively. Therefore, the density design variable $x_{e, 1}$ can be seen as a topological design variable as it chooses whether the element is void or solid. The remaining design variables are responsible for the material 
selection, given a pool of predefined materials.

The penalty $p$ plays an important role in penalizing intermediate density values, such that a discrete material distribution is obtained at the end of the design iterations. In Figure 1 the relation between the design variables (densities) and the interpolated material property, considering three phases (see Eq. 3), is plotted for different values of $p$. For $p=1$, the resulting interpolation is linear (plane), meaning that the intermediate densities are not penalized. Increasing the penalty factor leads to more concave surfaces, making it more uneconomical to have intermediate densities. Section 3.4 proposes a continuation approach as regards $p$ changes to promote escape from local minima.
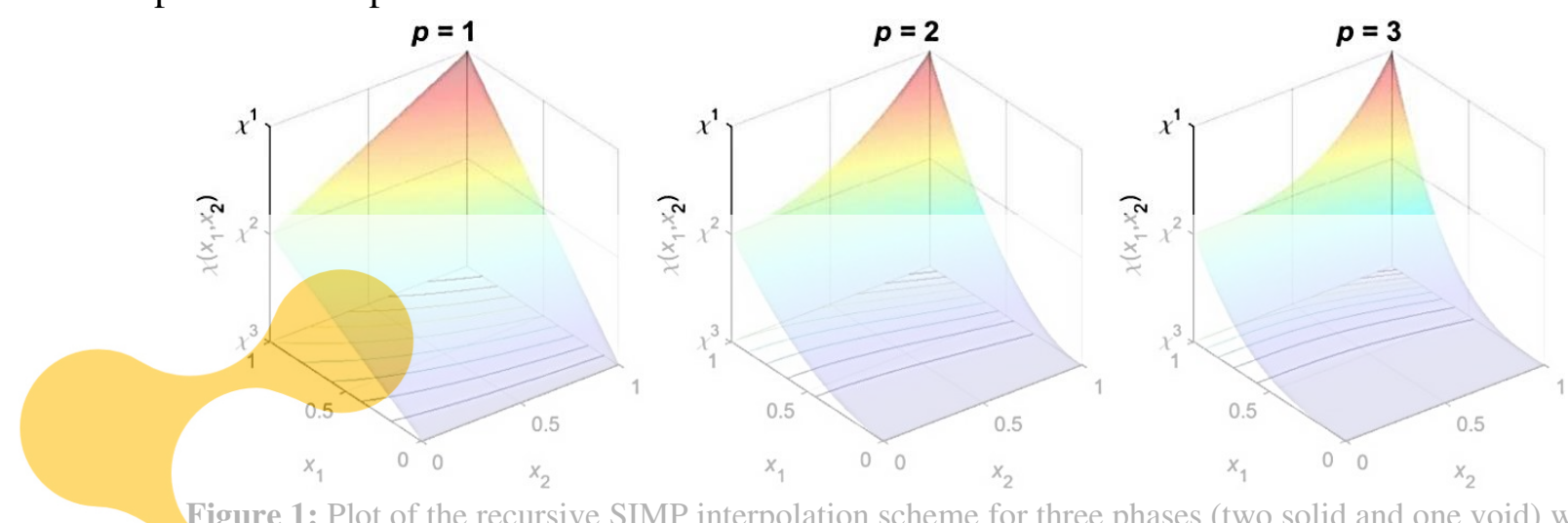

Figure 1: Plot of the recursive SIMP interpolation scheme for three phases (two solid and one void) with
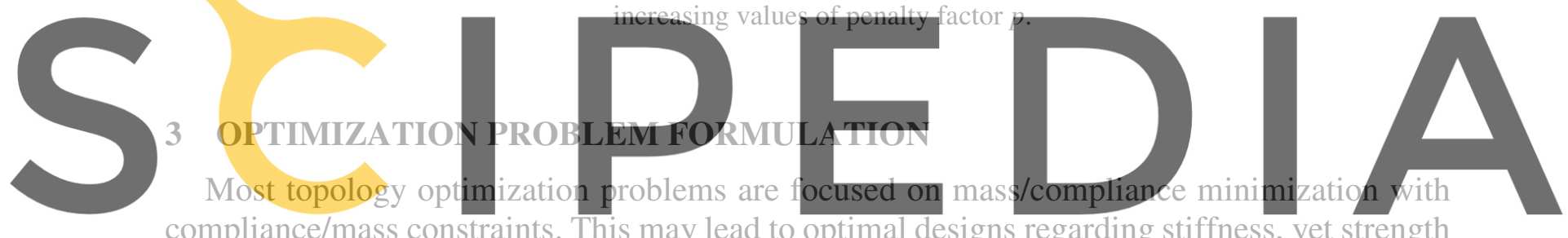

compliance/mass constraints. This may lead to optimal designs regarding stiffness, yet strength

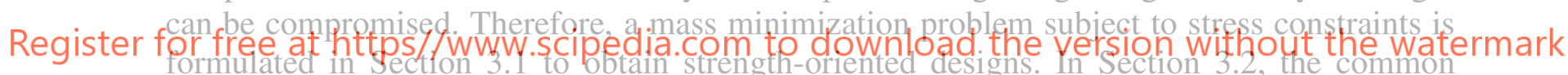
compliance minimization problem subject to mass constraint is revisited. These two formulations have the purpose of comparing the strongest and stiffest designs, respectively. The sensitivity analysis and penalty strategy are presented in Section 3.3 and 3.4, respectively. The problems are solved using the Method of Moving Asymptotes (MMA) [29].

\subsection{Mass minimization with stress constraints}

The mass minimization problem subject to stress constraints is formulated as:

$$
\min _{\mathbf{x}, x_{e, m} \in[\underline{x}, \bar{x}]} M(\mathbf{x})=\sum_{e=1}^{N E} \rho_{e}(\mathbf{x}) L_{e} A_{e}
$$

s.t.

$$
\left\{\begin{array}{l}
\mathbf{K u}=\mathbf{f}, \mathbf{K}=\sum_{e=1}^{N E} \mathbf{K}_{e}\left(E_{e}(\mathbf{x})\right) \\
g_{e}^{\sigma}(\mathbf{x}, \mathbf{u}(\mathbf{x}))=\left(\frac{\sigma_{e}(\mathbf{x}, \mathbf{u}(\mathbf{x}))}{\bar{\sigma}_{e}(\mathbf{x})}\right)^{2}-1 \leq 0 \\
g^{\eta}(\mathbf{x})=\frac{\psi(\mathbf{x})-\eta}{\eta} \leq 0, \text { with } \psi(\mathbf{x})=\sum_{e=1}^{N E} \sum_{m=1}^{N_{m a t}}\left[\left(\bar{x}-x_{e, m}\right)\left(x_{e, m}-\underline{x}\right)\right]
\end{array}\right.
$$


where $M(\mathbf{x})$ is the mass of the truss structure, $\mathbf{x}$ is the density variables vector, $\bar{x}$ and $\underline{x}$ are the respective upper $(=1)$ and lower (=0.001 to prevent singularities) bounds of $x_{e, m}, \bar{L}_{e}$ is the length and $A_{e}$ is the area, $g_{e}^{\sigma}$ is the normalized stress constraint of element $e$ and $g^{\eta}$ is the normalized intermediate density constraint, where $\eta$ is kept small enough. The total number of finite elements is $N E$, and $N_{\text {mat }}$ is the total number of solid phases, i.e., $n-1$. The volumetric mass density $\rho_{e}$, Young's modulus $E_{e}$ and yield stress $\bar{\sigma}_{e}$ are interpolated according to the scheme presented in Section 2, using different penalizations as presented in Section 3.4.

The stress in an element $e$ is computed through the material constitutive law:

$$
\sigma_{e}(\mathbf{x}, \mathbf{u}(\mathbf{x}))=E_{e}(\mathbf{x}) \varepsilon_{e}(\mathbf{u}(\mathbf{x}))
$$

where $\varepsilon_{e}$ is the strain of element $e$ that depends on the displacement field $\mathbf{u}$ which solves the equilibrium problem, $\mathbf{K u}=\mathbf{f}$, using a finite element formulation.

In order to overcome the stress singularity phenomenon, the $q p$-approach relaxation technique is used. This means that the exponent $p^{\bar{\sigma}}$ in the interpolation of the yield stress is made slightly lower than $p^{E}$ in the interpolation of the Young's modulus, i.e., $p^{\bar{\sigma}}<p^{E}$.

The compliance has not been included in (5), which is a simplification but its inclusion might be pondered to definitely exclude the trivial "no structure" solution. To proceed, if the penalization of intermediate densities is not on account of $p^{E}$, as it is in the case of compliance, the constraint $g^{\eta}$ ensures that one attains a discrete solution, i.e., densities are forced to attain their bounds, 0 and 1. A continuation approach for $p^{E}, p^{\bar{\sigma}}$ and $\eta$ is proposed in Section 3.4.
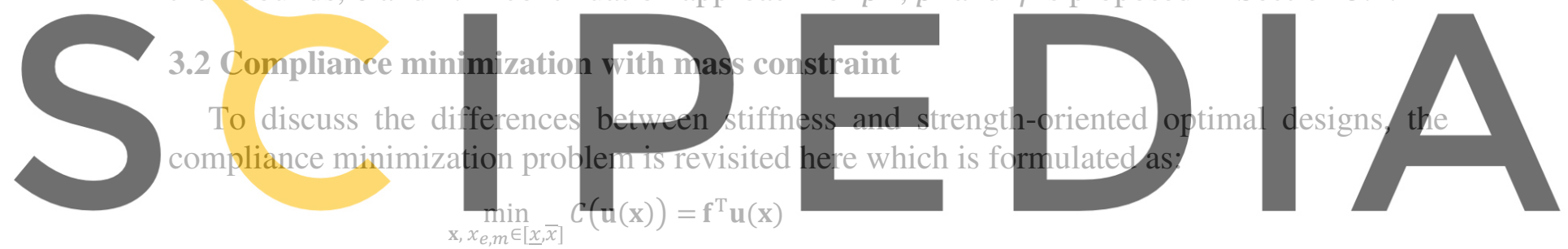

Register for free at https//NwwW.scipedia.com to download the version without the watermark

$$
\left\{\begin{array}{l}
\mathbb{K u}=\mathrm{f}, \mathbb{K}=\sum_{e=1}^{N E} \mathbb{K}_{e}\left(E_{e}(\mathbf{x})\right) \\
g^{M}(\mathbf{x})=\frac{M(\mathbf{x})}{M^{*}}-1 \leq 0 \\
g^{\eta}(\mathbf{x})=\frac{\psi(\mathbf{x})-\eta}{\eta} \leq 0, \psi(\mathbf{x})=\sum_{e=1}^{N E} \sum_{m=1}^{N_{m a t}}\left[\left(\bar{x}-x_{e, m}\right)\left(x_{e, m}-\underline{x}\right)\right]
\end{array}\right.
$$

where $C$ is the compliance, $M(\mathbf{x})$ is the structure's mass, and $M^{*}$ is the optimal mass obtained from problem (5) solution. Here, the intermediate densities are penalized using both $p^{E}>1$ and constraint $g^{\eta}$.

When the layouts of the compliance and stress problems differ, one notices that the density variables in the compliance problem have difficulty to strictly converge to their discrete values as the mass constraint $g^{M}$ tends to be activated. In fact, for binary variables, the mass of the stiffest structure from (7) may differ from the mass of the strongest structure from (5). By means of the formulation (7) one ensures that each density eventually becomes 0 or 1 . Finally, although stresses are not part of formulation (7), they are measured to compare with the stress distribution from (5). 


\subsection{Sensitivity analysis}

The sensitivities of the mass and intermediate densities constraints w.r.t. density design variables are:

$$
\begin{gathered}
\frac{\partial M(\mathbf{x})}{\partial x_{e, m}}=\frac{\partial \rho_{e}(\mathbf{x})}{\partial x_{e, m}} L_{e} A_{e} \\
\frac{\partial g^{\eta}(\mathbf{x})}{\partial x_{e, m}}=-\frac{2 x_{e, m}+\bar{x}+\underline{x}}{\eta}
\end{gathered}
$$

The stress constraints $g_{e}^{\sigma}$ and compliance $C$ have implicit design dependence, so one uses the adjoint method to analytically compute these derivatives. The compliance derivative is straightforward to compute, as it results in a self-adjoint problem, and it is given by [5]:

$$
\frac{\mathrm{d} C}{\mathrm{~d} x_{e, m}}=-\mathbf{u}^{\boldsymbol{\top}} \frac{\partial \mathbf{K}}{\partial x_{e, m}} \mathbf{u}=-\mathbf{u}^{\top} \frac{\partial E_{e}(\mathbf{x})}{\partial x_{e, m}} \frac{A_{e}}{L_{e}}\left[\begin{array}{rr}
1 & -1 \\
-1 & 1
\end{array}\right] \mathbf{u}
$$

Regarding the stress constraints $g_{e}^{\sigma}(\mathbf{x}, \mathbf{u}(\mathbf{x}))$, its sensitivities are computed through:

$$
\frac{\mathrm{d} g_{e}^{\sigma}(\mathbf{x}, \mathbf{u}(\mathbf{x}))}{\mathrm{d} x_{e, m}}=\frac{\partial g_{e}^{\sigma}}{\partial x_{e, m}}+\lambda_{e}^{\mathrm{T}}\left[\frac{\partial \mathbf{f}}{\partial x_{e, m}}-\frac{\partial \mathbf{K}}{\partial x_{e, m}} \mathbf{u}\right]
$$

where $\boldsymbol{\lambda}$ is the adjoint vector that solves the adjoint problem $\mathbb{K} \boldsymbol{\lambda}=\mathbf{z}=\left(\frac{\partial g_{e}^{\sigma}}{\partial \mathbf{u}}\right)^{\mathrm{T}}$. The required

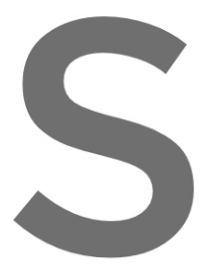
derivatives in (11) are
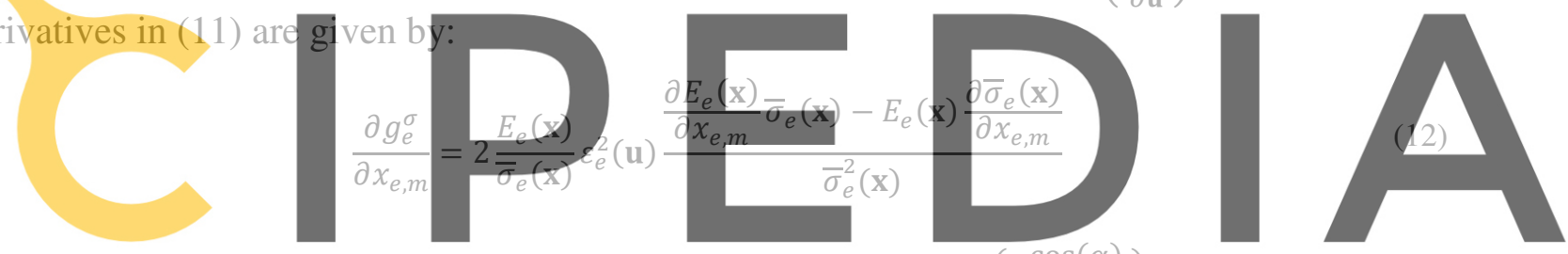

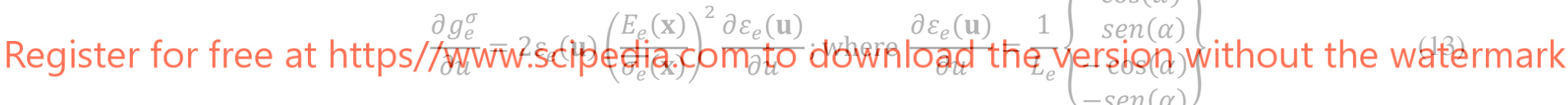

The load vector $\mathbf{f}$ is here considered independent from the design variables, therefore its derivatives are zero. The stiffness matrix $\mathbf{K}$ derivatives are computed as shown in (10). The $\alpha$ is the angle between the global (structure) and the local (element) coordinate systems.

The derivatives of the material properties of element $e\left(\rho_{e}, E_{e}\right.$ or $\left.\bar{\sigma}_{e}\right)$, simply involves differentiating (1) w.r.t. $x_{e, m}$.

\subsection{Penalization strategy}

To guarantee that the optimal solutions of problems (5) and (7) are discrete, i.e., all design variables are at their bounds ( 0 or 1$)$, and that a correct relaxation on stress constraints is applied, a penalization strategy is adopted. This penalization strategy, so-called continuation approach, is actually a twofold process, explained below, in order to prevent the algorithm from getting trapped in local minima.

In the first interval of design iterations, $I_{1}$, the values of the penalty exponents $p^{E}$ and $p^{\bar{\sigma}}$ are gradually increased according to Table 1 , from non-penalized values (that remain constant during the first $5 \%$ of $I_{1}$, see Fig. 2) to their maximum values at the end of $I_{1}$, remaining constant 
onward.

Right after the first interval of iterations, $I_{1}$, one has the interval of iterations defined as $I_{2}=$ $0.4 I_{1}$, where the value of the parameter $\eta$ is gradually decreased (see Table 1 ) such that, constraint $g^{\eta}$ becomes eventually active. Note that $\eta$ decreases to its minimum value of 0.001 after $70 \%$ of $I_{2}$ iterations have elapsed. Remark that, inside the interval $I_{1}$, the value of $\eta$ is made high enough on purpose such that the constraint $g^{\eta}$ is inactive. Finally, all parameters $p^{E}$, $p^{\bar{\sigma}}$ and $\eta$ remain constant during the last iterations allowing the algorithm to stabilise. The total number of iterations is then $I_{\text {total }}=I_{1}+I_{2}$. To better understand this continuation approach just described, please see Fig. 2.

Table 1: Initial and final values of some algorithm penalty parameters.

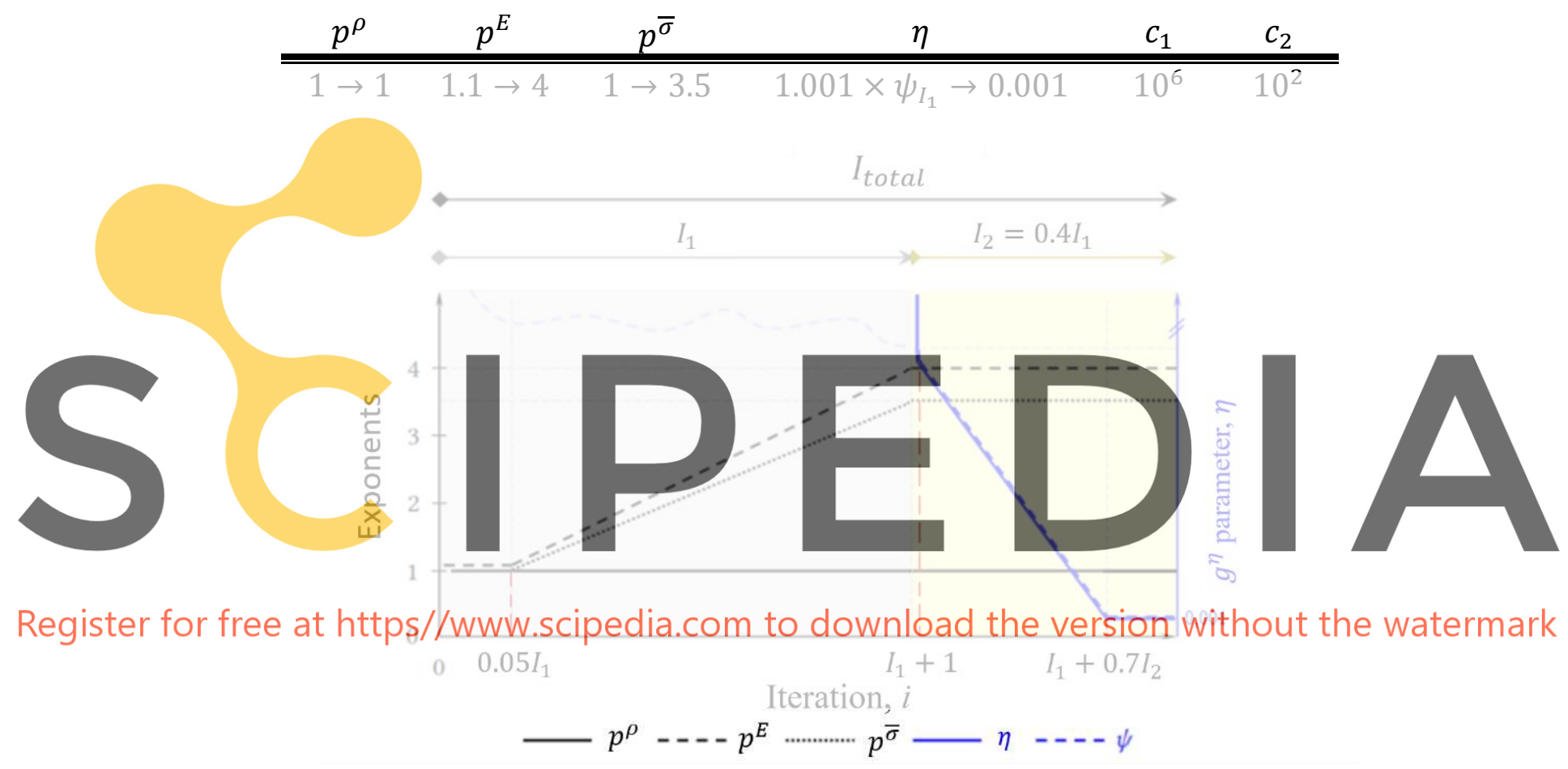

Figure 2: Penalization strategy: Variation of the penalty parameter values $\boldsymbol{p}^{\boldsymbol{\rho}}, \boldsymbol{p}^{\boldsymbol{E}}, \boldsymbol{p}^{\overline{\boldsymbol{\sigma}}}$ and $\boldsymbol{\eta}$ along the iterations.

The optimizer used here, MMA, uses artificial variables in subproblems that approximate the original problems, (5) or (7), such that feasibility is always attained. Each constraint has its own MMA penalty parameter, referred as $c_{1}$ for the stress/mass constraints and $c_{2}$ for the intermediate density constraint (see Table 1, last two columns). The tuning of the parameters presented in Table 1 is crucial. Preferably, these parameters should be as independent as possible of the numerical problem being solved.

\section{RESULTS}

In order to apply the proposed formulations, a 110 bar truss ground structure example is considered, see Fig 3. The bars have a circular cross-section area of $A=400 \times 10^{-6} \mathrm{~m}^{2}$, a length of $L=0.150 \mathrm{~m}$ and the load is $36.5 \mathrm{kN}$. Optimization problems are solved using MMA, 
implemented within a set of functions developed in MATLAB. Three different class of problems are considered, depending on the number of solid phases: (1) SMTO; (2) MMTO2 and (3) MMTO3. The properties of the solid phases are presented in Table 2.

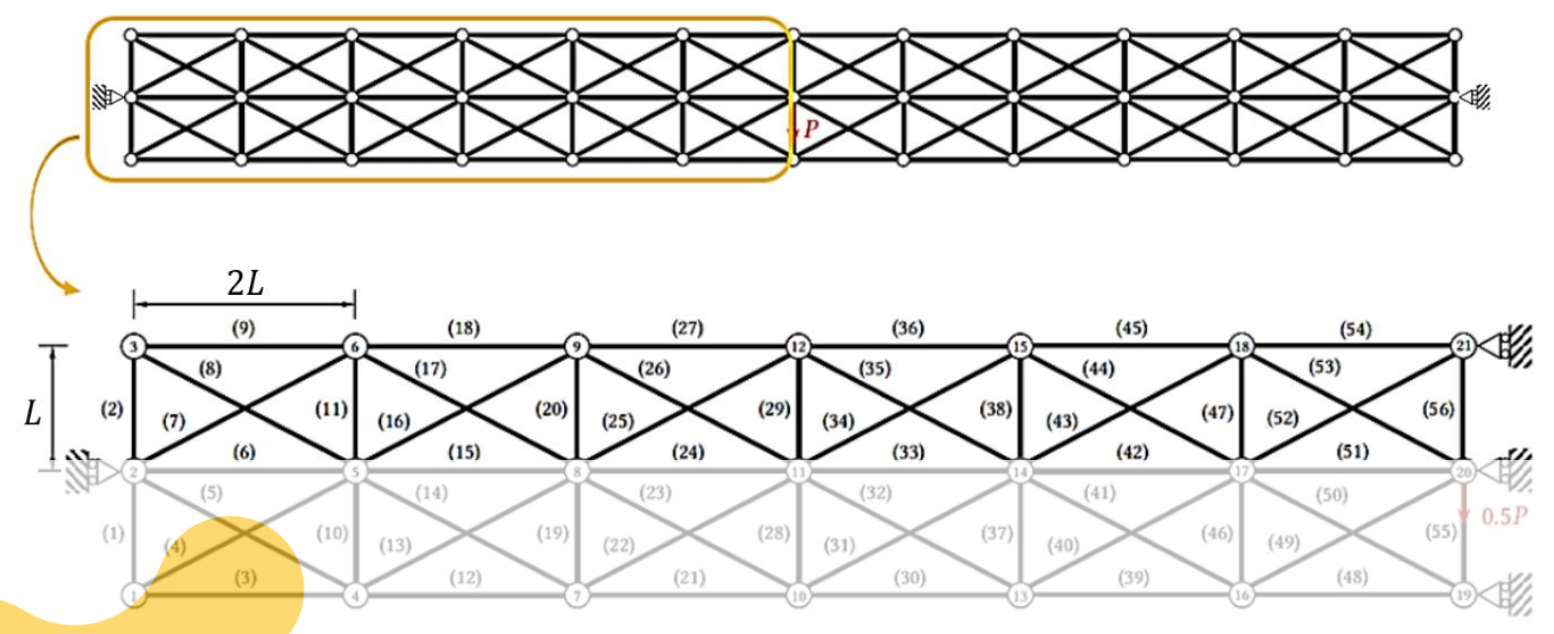

Figure 3: Ground structure of 110 truss bars (simplified to 56 bars due to symmetry conditions).
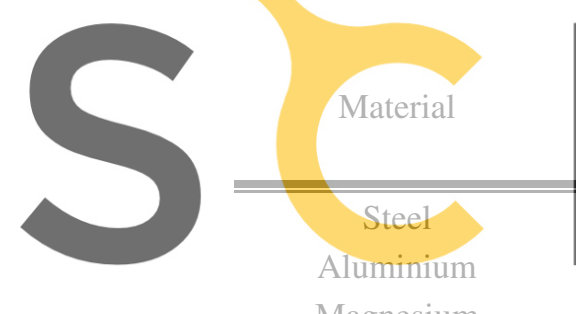

Magnesium

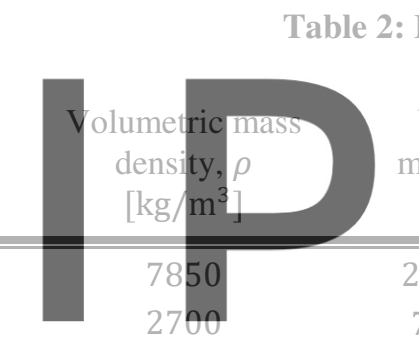

1700

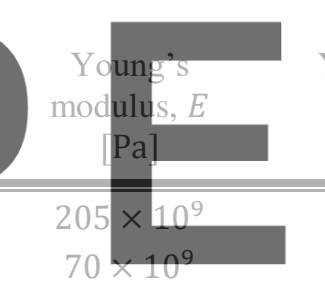

$44 \times 10^{9}$

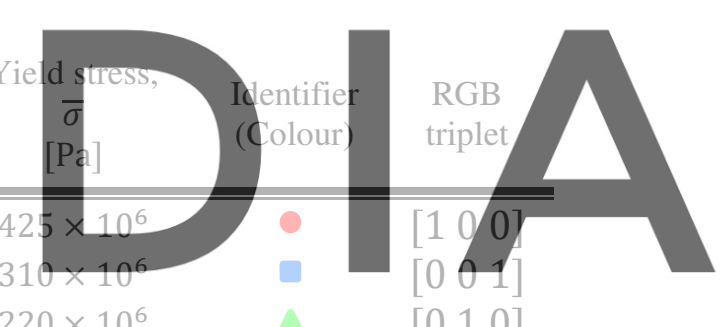

Register for free at https//www.scipedia.com to download the version without the watermark

\subsection{Mass minimization with stress constraints}

A density design variable $x_{e, m}$ for each solid phase and per finite element $e$ is required for proper interpolation. Therefore, the total number of design variables is obtained multiplying the total number of elements, $N E$, by the number of solid phases, $N^{\text {mat }}$, considered. As regards the number of constraints, it equals the total number of finite elements (number of stress constraints) plus one (the intermediate density penalization constraint), see (5).

Figure 4 shows two things for the SMTO, MMTO2 and MMTO3 problems solved. Firstly, the material distribution is seen on the left, where different colours represent different materials according to Table 2. Notice that hyperstaticity is obtained in every layout. Also, some connectivities indicate that a real truss bar groups two aligned bar finite elements from the original ground structure. Secondly, the normalized stress $\left(\sigma_{e} / \bar{\sigma}_{e}\right)$ at each bar element is seen on the right, and $\sigma_{e} / \bar{\sigma}_{e}=1$ means that the yield stress of the actual material is attained.

The values of the mass, maximum stress attained and compliance for each problem in Figure 4 are reported in Table 3. As shown, by adding aluminium to the structure (MMTO2) its mass can be reduced up to $48 \%$ and by adding magnesium (MMTO3) an additional mass reduction 
of $13 \%$ is achieved. Notice that the compliance $C^{M}$ is neither optimized nor constrained here. However, its value is evaluated for comparison purposes when solving the compliance-based problem, as presented next.

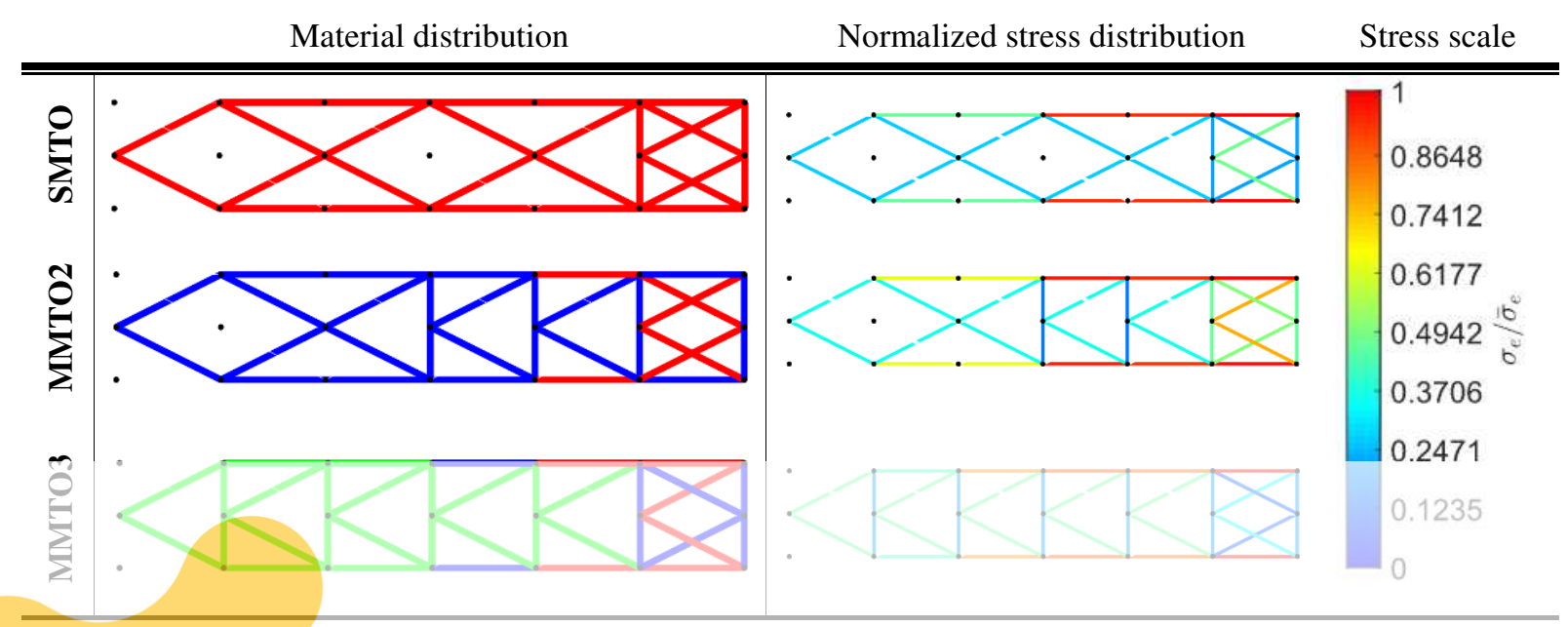

Figure 4: Optimal layout and material (on the left) and respective normalized stress (on the right) distributions for the SMTO, MMTO2 and MMTO3 problems minimizing mass with stress constraints.

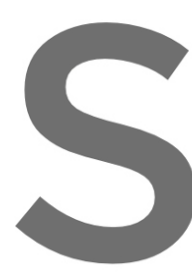

Table 3: Optimal objective function values, maximum stresses, compliances and design iterations for the

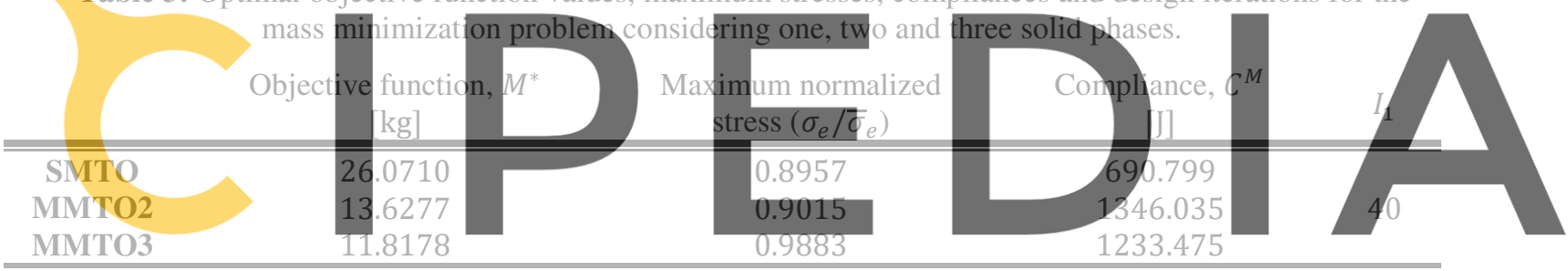

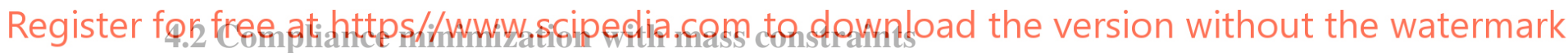

The compliance minimization problem also requires one density design variable $x_{e, m}$ for each solid phase and per finite element $e$. This problem is simpler than the previous one as it only requires two constraints, the mass and intermediate density penalization constraints. Figure 5 presents the optimized material distribution and the normalized stress $\left(\sigma_{e} / \bar{\sigma}_{e}\right)$, for the SMTO, MMTO2 and MMTO3 problems. The corresponding values of the compliance, maximum stress and mass are in Table 4.

Table 4, comparing to Table 3, presents better compliances $(6 \%, 34 \%$ and $17 \%$ less, respectively) but stresses are over the limit by $7,4 \%$ for approximately the same mass. In this Section stresses are evaluated (not constrained) for comparison purposes. The previous optimal mass values $\left(M^{*}\right)$, presented in Table 3 , are now the mass constraints upper bounds. However, one realizes from Table 4 that mass constraints are not actually active for minimum compliance, i.e., the obtained masses are slightly lower than $M^{*}$ values (compare third column of Table 4 with first column of Table 3). Since the strongest and stiffest layouts differ, the respective masses are hardly equal as densities take binary values ( 0 and 1$)$.

The stiffest and strongest designs are equivalent, as pointed out in [30], under specific conditions: single load case, uniform material, isostaticity, and unconstraint design variable, 
e.g. the cross section area variable. However, the problem presented here uses the artificial density as design variable, which has the side constraints 0 and 1 . Furthermore, the multimaterial case is considered here and the stress optimal trusses are statically indeterminate, which thus explains the difference between the strength and stiffness-oriented optimal designs.

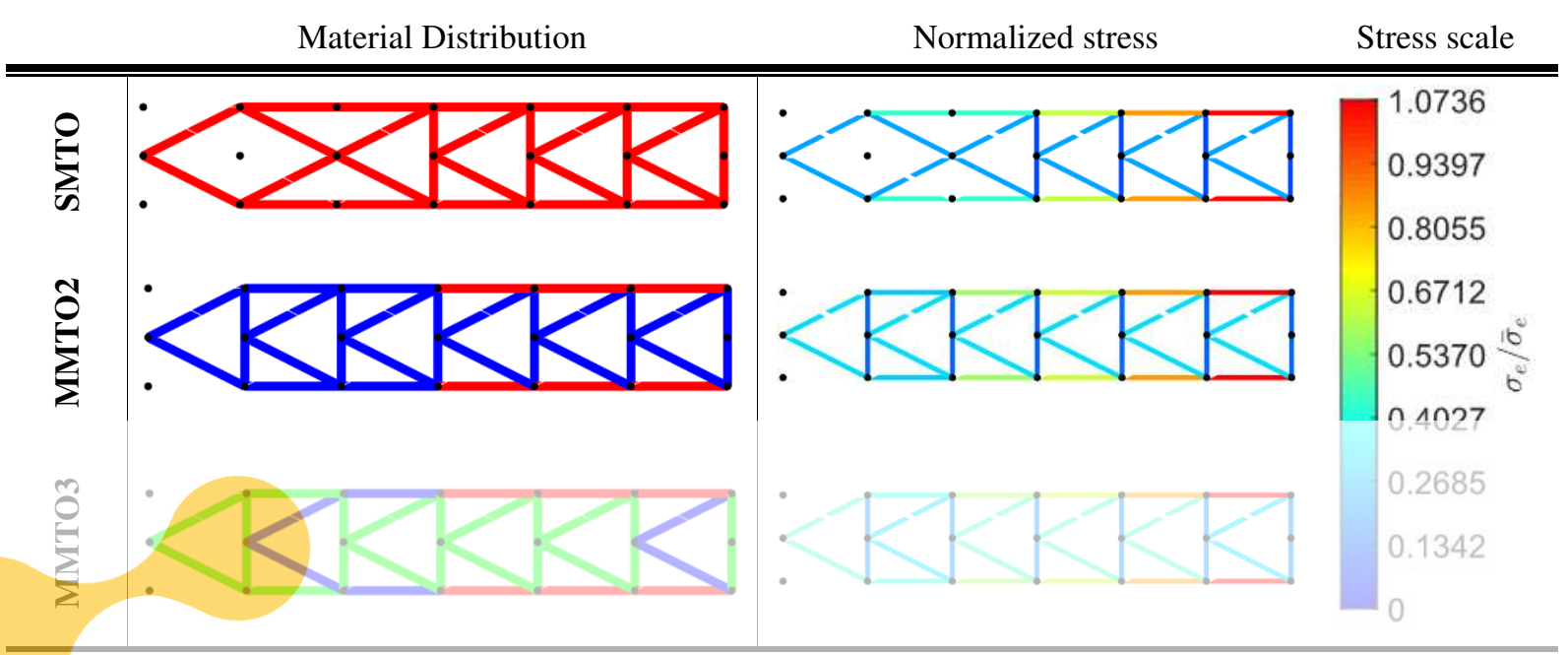

Figure 5: Optimal layout and material (on the left) and respective normalized stress (on the right) distributions for the SMTO, MMTO2 and MMTO3 problems minimizing compliance with mass constraint.

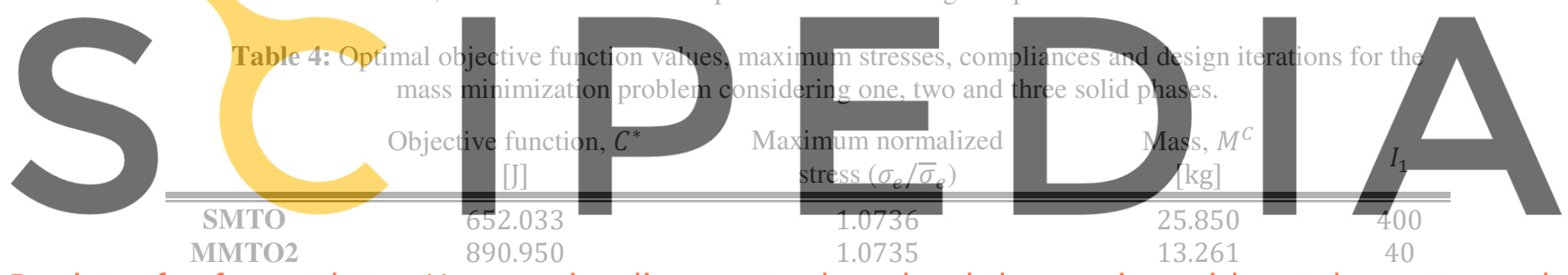

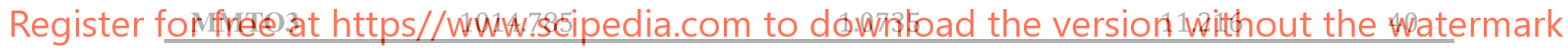

\section{CONCLUSIONS}

Stress-based TO problems are very important in engineering practice and not always the strength and stiffness-oriented designs coincide as seen in the present work. Performing TO without controlling the stresses in the structure can lead to optimal solutions that do not meet the material failure criterion, as seen in the compliance minimization problem in Section 4.2.

Usually when TO is applied to truss structures, the cross-sectional areas are used as design variables. However, keeping stresses under the yielding limit is not enough to prevent that thin members, under compression, appear with related buckling instability. Using artificial densities as design variables, considering pre-defined areas large enough, it might be seen here as a possible approach to avoid or minimize the buckling problems. The buckling problem is not an easy one to deal with. For instance, notice the buckling length jump when, at the final layout seen in Figure 4, a bar is identified grouping two aligned finite elements from the initial ground structure seen in Figure 3. This is the so-called chain effect that deserves careful treatment, out of the scope of the present work, yet not fully solved in the literature and thus can be addressed 
in the future.

The MMTO formulation proposed in this work, applied to trusses, uses a density-based approach. This approach allows a gradient-based optimizer to be used, since the problem is defined through differentiable functions that use continuous design variables. Considering more than one material in TO problems improves the performance in lightweight design when compared to the optimal single-material solution, as demonstrated in Section 4 with the example chosen.

To conclude, this work is a contribution on MMTO of truss structures including stress constraints. The numerical example provided shows the viability of the proposed formulations. As future work, the authors intend to include the areas as design variables (besides densities) as well as buckling constraints.

\section{ACKNOWLEDGMENTS}

Authors acknowledge Fundação para a Ciência e a Tecnologia (FCT - MCTES) for its financial support through the project UIDB/00667/2020 (UNIDEMI) and PhD scholarship SFRH/BD/136744/2018. Authors wish also to thank Professor Krister Svanberg (Royal Institute of Technology, Stockholm, Sweden) for the MMA optimization code.

\section{REFERENCES}

[1] Maxwell, J. C., On reciprocal figures, frames and diagrams of forces. Eding Roy Soc Proc (1870) 7:160-208.

[2] Dorn, W., Gomory, R., and Greenberg, M., Automatic design of optimal structures. $J$ Mec. (1964) 3:25-52.

[3] Hajela, P. and Lee, E., Genetic algorithms in truss topological optimization. Int. J. Solids Struct. (1995) 32:3341-3357.

[4] Stolpe, M., Truss optimization with discrete design variables: a critical review. Struct. Multidiscip. Optim. (2016) 53:349-374.

[5] Bendsøe, M. P. and Sigmund, O., Topology optimization - Theory, Methods and Applications. Springer, Berlin Heidelberg New York.

[6] Bendsøe, M. P. and Kikuchi, N., Generating optimal topologies in structural design using a homogenization method. Comput. Methods Appl. Mech. Eng. (1988) 71:197-224.

[7] Bendsøe, M. P., Optimal shape design as a material distribution problem. Struct. Multidiscip. Optim. (1989) 1:193-202.

[8] Zhu, J. H., Zhang, W. H., and Xia, L., Topology Optimization in Aircraft and Aerospace Structures Design. Arch. Comput. Methods Eng. (2016) 23:595-622.

[9] Zhong, W., Su, R., Gui, L., and Fan, Z., Multi-objective topology and sizing optimization of bus body frame. Struct. Multidiscip. Optim. (2016) 54:701-714.

[10] Sigmund, O. and Torquato, S., Design of materials with extreme thermal expansion using a three-phase topology optimization method. J. Mech. Phys. Solids (1997) 45:10371067.

[11] Gibiansky, L. V. and Sigmund, O., Multiphase composites with extremal bulk modulus. J. Mech. Phys. Solids (2000) 48:461-498.

[12] Stegmann, J. and Lund, E., Discrete material optimization of general composite shell structures. Int. J. Numer. Methods Eng. (2005) 62:2009-2027. 
[13] Guo, X., Zhang, W., and Zhong, W., Stress-related topology optimization of continuum structures involving multi-phase materials. Comput. Methods Appl. Mech. Eng. (2014) 268:632-655.

[14] Wang, Y., Gao, J., Luo, Z., Brown, T., and Zhang, N., Level-set topology optimization for multimaterial and multifunctional mechanical metamaterials. Eng. Optim. (2017) 49:22-42.

[15] Li, D. and Kim, I. Y., Multi-material topology optimization for practical lightweight design. Struct. Multidiscip. Optim. (2018) 58:1081-1094.

[16] Jung, Y., Lim, S., Kim, J., and Min, S., Lightweight design of electric bus roof structure using multi-material topology optimisation. Struct. Multidiscip. Optim. (2020) 61:12731285.

[17] Stankovic, T., Mueller, J., Egan, P., and Shea, K., A Generalized Optimality Criteria Method for Optimization of Additively Manufactured Multimaterial Lattice Structures. J. Mech. Des. (2015) 137:1-12.

[18] Zhang, X. S., Paulino, G. H., and Ramos, A. S., Multi-material topology optimization with multiple volume constraints: a general approach applied to ground structures with material nonlinearity. Struct. Multidiscip. Optim. (2018) 57:161-182.

[19] Kaveh, A. and Seddighian, M. R., Simultaneously multi-material layout, and connectivity optimization of truss structures via an Enriched Firefly Algorithm. Structures (2020) 27:2217-2231.

[20] Shintani, K., Chan, Y.-C., and Chen, W., Robust Multi-material Topology Optimization for Lattice Structure Under Material Uncertainties. Adv. Struct. Multidiscip. Optim. (2018) 1110-1123.

[21] Lu, S., Ma, H., Xin, L., and Zuo, W., Lightweight design of bus frames from multimaterial topology optimization to cross-sectional size optimization. Eng. Optim. (2019) 51:961-977.

[22] Shimoda, M. and Tani, S., Simultaneous shape and topology optimization method for frame structures with multi-materials. Struct. Multidiscip. Optim. (2021) .

[23] Rozvany, G. I. N. and Birker, T., On singular topologies in exact layout optimization. Struct. Optim. (1994) 8:228-235.

[24] Duysinx, P., Van Miegroet, L., Lemaire, E., Brüls, O., and Bruyneel, M., Topology and generalized shape optimization: Why stress constraints are so important? Int. J. Simul. Multidiscip. Des. Optim. (2008) 2:253-258.

[25] Kirsch, U., On singular topologies in optimum structural design. Struct. Optim. (1990) 2:133-142.

[26] Cheng, G. . and Guo, X., E-relaxed approach in structural topology optimization. Struct. Optim. (1997) 258-266.

[27] Duysinx, P. and Bendsøe, M. P., Topology optimization of continuum structures with local stress constraints. Int. J. Numer. Methods Eng. (1998) 43:1453-1478.

[28] Bruggi, M., On an alternative approach to stress constraints relaxation in topology optimization. Struct. Multidiscip. Optim. (2008) 36:125-141.

[29] K., S., The method of moving asymptotes - a new method for structural optimization. Int. J. Numer. Methods Eng. (1987) 24:359-373.

[30] Cheng, G., Some aspects of truss topology optimization. Struct. Optim. (1995) 10:173179. 